\title{
Clinicopathological profile of colorectal carcinoma in a tertiary care teaching hospital in Pokhara, Nepal.
}

\author{
Subash Bhattarai* (D), Om Bahadur Karki², Merina Gyawali³, Sudeep Regmi ${ }^{4}$ \\ I Department of Medicine, 2Department of surgery, 3Department of Radio diagnosis and Imaging, 4Department of Pathology, \\ Manipal College of Medical Sciences and Teaching Hospital, Pokhara, Nepal.
}

\begin{abstract}
Introduction: Colorectal cancer is the most common malignancy of the gastrointestinal tract. They are common after 50 years of age and present with bleeding per rectum, altered bowel habits, anemia, pain abdomen, and weight loss. The objective of this study was to evaluate the demographic profile, clinical presentation, colonoscopy and histopathology findings of colorectal cancer. Methods: A descriptive cross-sectional study was conducted. Cases were studied from the departmental records. Clinical presentation, CT abdomen, colonoscopy, and histopathology findings of colorectal carcinoma were studied. Data entry was done in SPSS version 20. Results: The mean age of subjects was $56 \pm 11.42$ years (range of $34-80$ years) with male predominance (M: $F=3: 2$ ). Changes in bowel habits (92.3\%), chronic abdominal pain $(84.6 \%)$, generalized weakness $(80.7 \%)$, rectal bleeding $(46.2 \%)$, and significant weight loss $(42.3 \%)$ were the most common presenting symptoms. Rectal carcinoma was the most common (35\%) followed by carcinomas of ascending colon (14\%) and descending colon (10\%). The most common morphology was proliferative growth (61.5\%). All colorectal carcinomas were adenocarcinoma, and the majority was moderately differentiated (51.9\%). Conclusion: Colorectal cancers are seen above 50 years of age and common in males. Changes in bowel habits, chronic abdominal pain, generalized weakness, rectal bleeding, and weight loss are the most common form of presentations. These patients usually present late with advanced disease and unfavorable histopathology. Screening colonoscopy $>50$ years is strongly recommended.
\end{abstract}

Key words: Clinical presentation, colorectal carcinoma, colonoscopy, histopathology

\section{*Correspondence:}

Dr Subash Bhattarai MBBS, MD, DM

Associate Professor, Unit of Gastroenterology, Department of Medicine,

Manipal College of Medical Sciences and Teaching Hospital, Pokhara, Nepal

Email: kiwisubash@yahoo.com

Submitted: September 1, 2020

Accepted: June 14, 2021

To cite: Bhattarai S, Karki O, Gyawali M, Regmi S. Clinicopathological profile of colorectal carcinoma in a tertiary care hospital in Pokhara, Nepal JGMC Nepal. 2021; 14(2):14-8. DOI: 10.3126/jgmcn.v14i1.30966

Mobile No: +9779815293117

\section{INTRODUCTION}

Colorectal cancer (CRC) is the most common malignancy in the gastrointestinal tract worldwide. CRC is the second most common cancer among women and the third most common among men. ${ }^{1}$ CRC is a multi-factorial disease. Genetic factors, environmental exposures, diet, and inflammatory conditions of the digestive tract are all involved in the development of colorectal carcinomas. ${ }^{2}$ These have varied clinical presentation like bleeding per rectum, altered bowel habits, anemia, pain abdomen, obstruction, and weight loss ${ }^{2,3}$ More than $90 \%$ of cancers occur after 50 years of age. ${ }^{2}$

Colonoscopy is an invasive procedure for diseases of the lower gastrointestinal tract. It is utilized for both diagnostic and therapeutic purposes. Direct visualization makes it an investigation of choice for various pathologies of colon. Polypectomy during colonoscopy has been shown to decrease the incidence of CRC and associated mortality. ${ }^{4}$ Patients who are not good candidates for colonoscopy can be evaluated using CECT (Contrast Enhanced Computed Tomography) abdomen and CT colonography. 
The incidence of CRC is increasing in developing countries including Nepal. ${ }^{5}$ This research is meant to study the demographic profile, clinical presentation, colonoscopy and histopathology findings of colorectal cancer at a tertiary care Teaching Hospital in Pokhara, Nepal.

\section{METHODS}

This cross-sectional descriptive, hospital based retrospective study was carried out in the unit of Medical Gastroenterology, Department of Medicine and Department of Surgery at Manipal College of Medical Sciences and Teaching Hospital, Nepal from January 2018 to June 2020 for a period of 30 months. Manipal Teaching Hospital is a tertiary care teaching hospital in Gandaki province, Nepal. The study was approved and verified by the Institutional Review (MEMG/IRC/383/GA).

Cases were studied from the departmental records including admission and discharge summaries. All consecutive cases of 18 years and above that presented with varied lower gastro intestinal (GI) complaints either as out-patients or in-patients and subsequently diagnosed with colorectal carcinoma were enrolled for the study. Patients with no histopathological confirmation or those with incomplete records were excluded from the study. Data regarding demographic variables, clinical presentation and complications, histopathological correlations were studied and alongside blood investigations like complete blood count, platelets count, blood grouping, liver function test, prothrombin time / international normalized ratio (PT / INR), coagulation profile, viral serologies and tumor markers like CEA were obtained from patients' records and files. Data collected were entered in a preformed sheet. CECT (Contrast Enhanced Computed Tomography; PHILIPS Ingenuity 128 CT Scanner, NETHERLANDS Inc) scan of the abdomen and pelvis was done for a complete evaluation of lower GI tract and assessing enlarged regional lymph nodes, ascites, and metastases.

After hemodynamic stabilization, bowel preparation, and pre-medications and informed consent, colonoscopy (PENTAX EPK 700, PENTAX JAPAN Inc) was performed. Colonoscopy findings were noted, and biopsy of tissue was sent for histopathological study. Clinical, radiological, colonoscopy findings and histopathology were helpful in making the final diagnosis.

Data was entered in Microsoft Excel 2010 and analysis was done using SPSS version 20. All categorical data were expressed in percent and absolute number. All numerical continuous data were expressed in mean \pm SD. Chi squared test was used to compare difference of proportions for categorical data. All tests were analyzed with a 95\% confidence interval and results were considered significant if $P$ value $<0.05$.

\section{RESULTS}

The departmental records showed a total of 71 patients with colorectal carcinoma during the study period of 30 months. Twelve patients had refused further evaluation with colonoscopy and histological confirmation and seven patients had incomplete records. So, finally 52 cases with clinical, radiological, colonoscopy findings suggestive of and histopathological confirmation of colorectal carcinoma were taken up for the study. The study group comprised of $32(61.5 \%)$ males and 20 (38.5\%) females (M: F=3:2). The mean age of subjects was $56 \pm 11.42$ years (range of 34 to 80 years). Three (5.8\%) patients were aged below 40 years of age. Patients were further classified as per sex and age groups (Table 1$)$. Majority $(n=41 ; 78.9 \%)$ of cases were aged between 51-70 years of age. There was no any gender difference in occurrence of CRC among different age groups statistically (Chi sq. test statistic=1.365; $\mathrm{df}=2$; $\mathrm{P}=0.56$ )

Table 1. Age groups/sex distribution of patients $(n=52)$

\begin{tabular}{lccc}
\hline Age group (years) & Males & Females & Total \\
$31-50$ & 5 & 1 & 6 \\
$51-70$ & 24 & 17 & 41 \\
$>71$ & 3 & 2 & 5 \\
Total & 32 & 20 & 52 \\
\hline
\end{tabular}

Change in bowel habits in 48(92.3\%) and chronic abdominal pain in $44(84.6 \%)$ were the most common presenting symptoms of the patients (Table 2).

Table 2: Presenting symptomatology of study patients $(n=52)$

\begin{tabular}{lcc}
\hline Symptomatology & Number & Percentage \\
Change in bowel habits & 48 & 92.3 \\
Chronic abdominal pain & 44 & 84.6 \\
Generalized weakness & 42 & 80.7 \\
Vomiting & 36 & 69.2 \\
Chronic diarrhea & 26 & 50 \\
Constipation & 24 & 46.2 \\
Rectal bleeding & 24 & 46.2 \\
Significant weight loss in last 6 months & 22 & 42.3 \\
Hemorrhoids & 9 & 17.3 \\
Abdominal swelling & 8 & 15.4 \\
Intestinal obstruction & 6 & 11.6 \\
Anemia of unknown origin & 7 & 13.5 \\
Jaundice & 6 & 11.5 \\
\hline
\end{tabular}

Among the clinical signs, pallor was the most common 
finding in 39(75\%), followed by abdominal tenderness in 36(69.2\%), and palpable abdominal mass in 13(25\%). Mean hemoglobin at presentation was 9.4 gm\%. ESR was raised in $43(82.7 \%)$ patients. Liver function tests revealed hyperbilirubinemia in nine $(17.3 \%)$ patients. Tumor marker carcinoembryonic antigen (CEA) was elevated (more than $4 \mathrm{ng} / \mathrm{ml}$ ) in 39 (75\%) patients.

CECT of the abdomen revealed findings suggestive or suspicious of colorectal malignancies in 39(75\%) patients. Majority (46.2\%) of these patients presented with Stage III carcinoma followed by stage II (25.6\%), stage IV (15.4\%) and Stage I (12.8\%). Four (7.7\%) patients had metastases in the liver with unknown primary. However, colon appeared normal in CECT in these four patients.

Rectal carcinoma was the commonest colorectal carcinoma and seen in 18 (35\%) patients followed by carcinoma of ascending colon in 10 (14\%) and descending colon in eight (10\%) patients (Bar diagram 1). Recto-sigmoid comprised of $24(46.2 \%)$ colorectal carcinomas. Almost $2 / 3^{\text {rd }}(65.4 \%$; 34 out of 52) of colonic carcinoma were left sided. Colorectal carcinoma was observed as either proliferative growth $(\mathrm{n}=32 ; 61.5 \%)$ or ulcers $(\mathrm{n}=10 ; 19.2 \%)$ or infiltrative $(\mathrm{n}=6$; $11.6 \%)$ or polyps $(n=4 ; 7.7 \%)$ on colonoscopy.

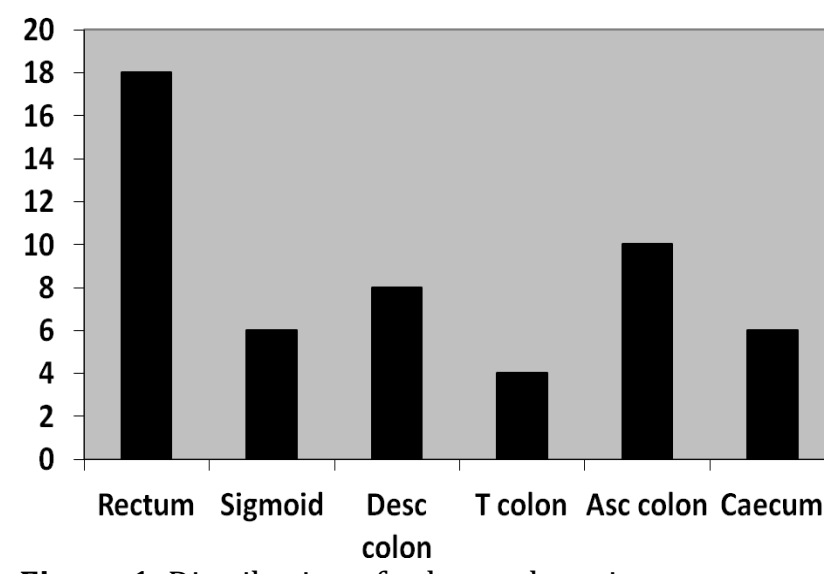

Figure 1: Distribution of colorectal carcinoma

(Desc colon=Decending colon, $\mathrm{T}$ colon=Transverse colon, Asc colon=Ascending colon)

All these 52 cases of colorectal carcinoma were histological proven. All were adenocarcinoma. Fourteen (26.9\%) were well differentiated, 27(51.9\%) were moderately differentiated and 11 (21.2\%) were poorly differentiated.

\section{DISCUSSION}

The mean age of subjects was $56 \pm 11.42$ years with male predominance (M: $\mathrm{F}=3: 2)$. Majority (78.9\%) of cases were aged between 51-70 years of age. There was no any gender difference in occurrence of CRC among different age groups. These findings were consistent with the study by Bhat et al. $^{6}$ in Kashmir India. The mean age of presentation was 54.7 years in a multicenter study comprising of 1525 patients at Colombia ${ }^{7}$. In a similar study by Paudyal et al. ${ }^{8}$ in Nepal and Kumar et al. ${ }^{9}$ in Oman, the mean age was 56.9 and 56 years respectively with male predominance.

In the current study, $4.8 \%$ patients were aged below 40 years of age. In a previous study in Nepal by Paudyal et al. $^{8}$, the incidence of colorectal cancer in young below 40 years of age was even higher (16.7\%). Colorectal carcinomas were detected at even lower ages (less than 35 years) among $19.25 \%$ patients by Bhat et al. ${ }^{6}$ Bohorquez et al. $^{7}$ had also reported $26.5 \%$ of cases diagnosed with CRC before the age of 50 years. So colorectal carcinoma nowadays, is not only restricted to age beyond 50 years. It is not uncommon in younger age.

Change in bowel habits (92.3\%), chronic abdominal pain $(84.6 \%)$, generalized weakness $(80.7 \%)$, vomiting (69.2\%), per rectal bleeding (46.2\%), and significant weight loss in last six months (42.3\%) were the most common presenting symptoms in the current study. Similar were the presentation according to Bhat et al. ${ }^{6}$ Abdominal pain was commonest presentation (86\%), followed by per rectal (PR) bleeding (55\%) according to Paudyal et al. ${ }^{8}$ Among the clinical signs, pallor was the most common finding $(75 \%)$ in the current study. Pallor was evident in only $52 \%$ patients in the study by Bhat et al. ${ }^{6}$

Tumor marker carcinoembryonic antigen (CEA) was elevated (more than $4 \mathrm{ng} / \mathrm{ml}$ ) in $75 \%$ patients in the current study. CEA level was elevated in lesser patients, $64.2 \%$ and $44.9 \%$ in the studies by Paudyal et al. ${ }^{8}$ and Bhat et al. $^{6}$ respectively. So, all these studies suggest that cases of CRC can exist in up-to $1 / 3^{\text {rd }}$ to $1 / 4^{\text {th }}$ of patients despite normal CEA levels.

Rectal carcinoma was the commonest (35\%) followed by carcinoma of ascending colon (14\%) and descending colon $(10 \%)$ in the current study. These findings were in consistent with Bhat et al. $^{6}$ where rectum (44\%) was the commonest site followed by Ascending Colon (29\%) and Descending Colon (12\%). Study by Kumar et al. ${ }^{9}$ also suggests that rectum (29.6\%) was the most common site of colorectal cancers followed by ascending colon.

Recto-sigmoid comprised of $46.2 \%$ of colorectal carcinomas in the current study. Recto-sigmoid involvement was 54\% and $63.8 \%$ according to Paudyal et al. ${ }^{8}$ and Bhat et al. ${ }^{6}$ respectively. In the current study, 2/3rd (65.4\%) of colonic 
carcinoma were left sided. Whereas, the incidence of left sided colorectal cancers were even higher $(72 \%$ and $75 \%)$ according to Paudyal et al. ${ }^{8}$ and Crenad et al. ${ }^{10}$ respectively.

In this study, colorectal carcinoma was observed as either proliferative growth $(53.8 \%)$ or ulcers $(26.9 \%)$ or infiltrative $(11.6 \%)$ or polyps $(7.7 \%)$ on colonoscopy. Proliferative $(41.5 \%)$ was similarly the commonest tumor morphology according to Bhat et al. ${ }^{6}$ Whereas, ulcerative (37.8\%) was more common morphology followed by infiltrative (33.7\%) and proliferative (23.7\%) according to Paudyal et al. $^{8}$

Majority (46.2 \%) of these patients presented with Stage III carcinoma followed by stage II (25.6\%), stage IV (15.4\%) and Stage I (12.8\%) in this study. Patients presented most commonly with Stage III (49.8\%) in the study by Bhat et al. ${ }^{6}$ in India. Amin et al. ${ }^{11}$ in Saudi Arabia found that majority (36\%) of patients presented in stage IV followed by $30 \%$ in stage III, $23 \%$ in stage II, and $11 \%$ in stage I. Patients had stage III (42.6\%) and stage IV (32.7\%) disease according to Kumar et al. ${ }^{9}$ in Oman. All these studies suggest that patients with colorectal carcinomas usually present late and with advanced stage.

In this study, all colorectal carcinomas were histologically adenocarcinoma. The most common morphology was moderately differentiated (51.9\%) followed by well differentiated (26.9\%) and poorly differentiated (21.2\%). Similar were the findings in a previous study in Nepal by Paudyal et al. $^{8}$ where all cases of CRC were adenocarcinoma, and majority (70\%) of them were of moderate differentiation. Histologically, 99\% patients had adenocarcinoma, and $49.7 \%$ of them were well differentiated according to Bhat et al. $^{6}$ Rest, $34.2 \%$ were moderately differentiated and $16.1 \%$ were poorly differentiated in that study. All these studies suggest that patients with colorectal adenocarcinomas usually present with advanced morphology in histopathology.

This study had its own limitations. It was a retrospective study. Operability and clinical outcomes were not studied. The study reflects to the findings of a specific geographical area with a small sample size.

\section{CONCLUSION}

Colorectal cancers were seen above 50 years of age and commonly in males. Change in bowel habits, chronic abdominal pain, generalized weakness, rectal bleeding and weight loss were the most common form of presentations. Rectum was the most common site and majority of them presented with proliferative growth on colonoscopy and CECT abdomen. These patients presented late with local spread and metastases. Majority had moderately differentiated adenocarcinoma in histopathology.

\section{Funding}

No Funding was received for the conduction and publication of the study.

\section{Conflict of interest}

Authors declare no conflict of interest.

\section{REFERENCES}

1. Ferlay J, Shin HR, Bray F, Forman D, Mathers C, Parkin DM. Estimates of worldwide burden of cancer in 2008: GLOBOCAN 2008. Int J Cancer. 2010; 127(12):2893917.

2. Boyle P, Langman JS. ABC of colorectal cancer: epidemiology. BMJ.2000; 321(7264):805-8.

3. Larsen IK, Bray F. Trends in colorectal cancer incidence in Norway 1962-2006: an interpretation of the temporal patterns by anatomic subsite. Int J Cancer. 2010;1 26(3):721-32. DOI: 10.1002/ijc.24839

4. Winawer SW, Fletcher RH, Mille L, Godlee F, Stolar $\mathrm{MH}$, Mulrow $\mathrm{CD}$, et al. AGA guidelines: Colorectal cancer screening: Clinical guidelines and rationale, Gastroenterology.1997;112(2): 594-642. DOI: 10.1053/gast.1997.v112.agast970594 PMID: 9024315.

5. Pradhananga KK, Baral M, Shrestha BM. Multiinstitution hospital-based cancer incidence data for Nepal - an initial report. Asian Pac J Cancer Prev. 2009; 10(2):259-62. PMID: 19537894.

6. Bhat SA, Chowdri NA, Khan MA, Parray FQ, Wani RA, Mehraj A, et al. Clinicopathological profile of colorectal cancer in Kashmir. Clin Surg. 2019;4:2368.

7. Bohorquez M, Sahasrabudhe R, Criollo A, SanabriaSalas MC, Vélez A, Castro JM, et al. Clinical manifestations of colorectal cancer patients from a large multicenter study in Colombia. Medicine (Baltimore). 2016; 95(40): e4883 DOI: 10.1097/ MD.0000000000004883 PMID: 27749544.

8. Paudyal S, KC SR, Maharjan S, Shah S, Giri N, KC S, et al. Clinicopathological profile of colorectal cancer managed at a university teaching hospital, Nepal. Journal of Patan Academy of Health Sciences. 2019; 6(2):31-8. DOI: 10.3126/jpahs.v6i2.27227

9. Kumar S, Ikram AB, Zahid KF, D Souza PC, Belushi MA, MuftiTD, etal.Colorectal cancer patientcharacteristics, 
treatment and survival in Oman - a single center study. Asian Pac J Cancer Prev. 2015; 16(12):485358. DOI:10.7314/apjcp.2015.16.12.4853. PMID: 26163603.

10. Crerand S, Feeley TM, Waldron RP, Corrigan T, Hederman W, O'Connell FX, et al. Colorectal carcinoma over 30 years at one hospital: no evidence for a shift to the right. Int J Colorectal Dis. 1991; 6(4):184-7.
11. Amin TT, Suleman W, Taissan AA, AlJoher AA, Mulhim OA, Yousef AH, et al. Patients' profile, clinical presentations and histopathological features of colorectal cancer in Al Hassa Region, Saudi Arabia. Asian Pacific J Cancer Prev. 2012; 13(1):211-6. DOI: 10.7314/APJCP.2012.13.1.211 